Radiotherapy

\section{Radiotherapy and intracranial meningiomas causing visual disturbance}

J O'Day

\section{Grounds for cautious optimism in the treatment of this condition}

$\mathrm{T}$ he meningiomas account for approximately $15 \%$ of intracranial tumours. Most meningiomas are benign slow growing well circumscribed tumours. The preferred mode of treatment is total surgical excision unless total removal would result in an unacceptably high morbidity. ${ }^{1}$ Certainly there have been changes to neurosurgical techniques with operative stereotaxy integrated with preoperative imaging to delineate more clearly the extent of the tumour and with it the ability to remove the lesion with less damage to surrounding structures.

However, if there is incomplete resection of the tumour then the cumulative risk of progression increases slowly over $10-15$ years to $90 \% .^{2}$ Radiotherapy is the one technique used as adjuvant therapy that has been shown consistently to slow the rate of recurrence and sometimes to partially reverse visual loss when present.

Radiotherapy, however, has side effects as it may lead to primary axonal damage or secondary axonal damage from vascular occlusion.

Recently developed radiotherapy techniques have been introduced to try and limit the radiotherapy treatment as much as possible to the tumour and not to the surrounding tissues and

$\mathrm{ROP}$ fractionated stereotactic radiotherapy is one such modality.

Behbehani et al in this issue of $B J O$ (p 130) have reported the use of fractionated stereotactic radiotherapy (FSRT) for treating parasellar meningiomas. This is a retrospective series of 13 patients and the authors have shown that in $75 \%$ of in $57 \%$ of eyes the visual field improved. There was a diminution in central acuity in $12.5 \%$ and a decrease in visual field in $15 \%$ of eyes. This is the first significant series of patients with parasellar meningiomas with visual field loss, who have undergone this form of radiotherapy, to be reported.

There has already been a number of published series using the same technique with optic nerve meningiomas where there has been a stabilisation in vision and occasionally a visual improvement.

There are, however, some questions that remain unanswered. A dose of 50.4 Gy was given in 28 fractions over a 5 week period. The ideal dose however for radiation treatment is not known precisely because of individual tolerance of the varying dose that will be administered to surrounding structures. The general guideline is that $60 \mathrm{~Gy}$ in divided fractions causes a 5\% incidence eyes the visual acuity remains stable and

\title{
Retinopathy of prematurity's turning point
}

\section{W V Good, R L Gendron}

This point may be identified ophthalmoscopically as the subthreshold (prethreshold) state for some eyes

Stepp'd in so far that, should I wade no more,

Returning were as tedious as go o'er. (William Shakespeare (1564-1616) Macbeth. Act iii, Sc 4)
A s new and successful treatments emerge for the management of it becomes increasingly apparent that considerable morbidity accompanies of neurotoxicity. However, the problems with irradiation, particularly in slowly dividing tissues such as the central nervous system, may not be manifest for a number of years and so although there are grounds for cautious optimism from this reported series, I think that long term effects of fractionated stereotactic radiotherapy will be important to report.

Certainly the authors do report the recent article where radiation retinopathy had occurred 22 months after FSRT optic nerve sheath meningioma.

Unfortunately, it is difficult to obtain the series of patients that is large enough to provide statistically significant results. However, a European group is currently designing a prospective meningioma randomised trial of observation versus conventionally fractionated radiotherapy or radiosurgery after non-radical surgery in benign intracranial meningioma. This study will use various radiotherapy techniques and perhaps will help to further elucidate the controversy over the efficacy and side effects of radiotherapy treatment for meningioma.

This paper by Behbehani et al does, however, give us grounds for cautious optimism in the treatment of this condition and I encourage them to report on this group of patients again when there has been a longer lapse of time.

Br J Ophthalmol 2005;89:127.

doi: 10.1136/bjo.2004.055525

Correspondence to: J O'Day, Victoria Parade Eye Consultants, 55 Victoria Parade, Fitzroy, Victoria 3065, Australia; justinoday@

bigpond.com

\section{REFERENCES}

1 Mirimanoff RO. New radiotherapy technologies for meningiomas. Radiother Oncol 2004:71:247-9.

2 Mirimanoff RO, Dosoretz D, Linggood R, et al. Meningioma: analysis of recurrence and progression following neurosurgical resection J Neurosurg 1985;62:18-24.

more advanced disease, even when the condition regresses, and with or without treatment to effect regression. ROP research perforce will be aimed at further reducing blindness from the disease, and also at eliminating these other complications.

What are these other complications? Myopia is one, but unfavourable optotype acuity, strabismus, anisometropia, amblyopia, glaucoma, and cataract can all occur in the absence of retinal detachment. The paper by Sahni and associates in this issue of the $B J O$ ( $p$ 154) reminds us that infants with regressed, prethreshold ROP eyes have better visual acuity, refractive error outcome, and anatomical outcome than infants whose ROP progresses to require treatment. Somewhere in the disease process 
a point is reached, beyond which there is no turning back. The authors should be congratulated on their thought provoking effort. Since this work confirms findings in other experiments, it begs the question, what is it about more advanced disease that creates these problems? When or where in the disease process do complications become likely, and how does this happen?

Basic science findings in ROP research offer some explanation. Molecular events in the ROP disease process trigger changes in the developing eye, which extend beyond the mechanical events leading to retinal detachment. A placentally derived protein, insulin-like growth factor (IgF-1), affects the production of vascular endothelial growth factor $\left(\right.$ VEGF) ${ }^{1}$ and this latter growth factor no doubt is responsible for neovascularisation seen in stage 3 ROP. Supporting this is the finding from in situ studies, demonstrating upregulation of VEGF in areas adjacent to neovascularisation. Neovascularisation may lead to cicatricial changes and, in the worst cases, traction retinal detachments.

Exactly when in the development of ROP that VEGF and its molecular control pathways are induced needs investigating. Indeed, molecular events determining VEGF levels are complex and might even be occurring well before VEGF levels reach a detrimental point in ROP. Molecular processes controlling VEGF are only now being elucidated. HIF- $1 \alpha$ regulates VEGF expression during hypoxia and can promote blood vessel proliferation through its induction of VEGF. ${ }^{2}$ Acetylation and ubiquitin targeted degradation of HIF- $1 \alpha$ may be a key control point for VEGF expression in mammals. ${ }^{3}$ Early molecular events such as these occurring in the retina might promote the stabilisation of hypoxia inducible factor-lalpha (HIF-l $\alpha$ ) and the production of growth factors promoting neovascularisation in the retina.

While VEGF is a growth factor, there are also homeostatic proteins which could be important to the disease process. One such protein is Tubedown-1, present in stable retinal vascular beds. Its downregulation has been demonstrated in proliferative diabetic retinopathy. ${ }^{4}$ Recent studies in transgenic animals have established that loss of Tubedown-1 leads to neovascular proliferative retinopathy. ${ }^{5}$ It is conceivable that this and other homeostatic proteins could have a role in development of neovascularisation in ROP and possibly in the severity of ROP disease outcome.
In Sahni et al's paper, the turning point in the ROP disease process is heralded by the development of threshold disease, but the molecular turning point and effects of VEGF and other proteins extend beyond these clinical vascular findings. Molecular events surely determine the development of myopia and reduced visual acuity. Eyes with spontaneous regression of subthreshold disease show less myopia and more favourable acuity outcomes.

The authors raise additional intriguing questions with their findings. One of these concerns the effectiveness of laser surgery compared with cryosurgery for advanced ROP disease. Another finding, that there is less myopia after laser treatment than after cryosurgery, is also interesting. Despite the authors' findings, the benefit of laser versus cryotherapy remains an open question, in our opinion. Baseline characteristics for infants in this study indicate that infants in the various groups may have differed in significant ways. Regressed subthreshold eyes occurred in larger birthweight infants; laser treated eyes also occurred in larger birthweight infants, compared with infants who had cryosurgery treated eyes. Enrolment into the study occurred at $<1500 \mathrm{~g}$, whereas enrolment in many other larger studies occurred in infants $<1251 \mathrm{~g}$. The study is retrospective, and selection bias could have crept into the management of infants with ROP. Laser replaced cryosurgery in the past 15 years in many centres and, simultaneously, advances in neonatal care may have affected retinal outcomes in premature infants.

Also of note is the finding that strabismus is associated with intraventricular haemorrhage (IVH) and anisometropia. We are reminded that vision problems may result from neurological injury, also more common in very premature infants. Some children with favourable retinal outcomes will still see poorly because of neurological damage. It would be helpful to identify the incidence of neurologically based vision impairment.

At a point in the life of some premature infants, a line of no return is crossed, and eye injury ensues. Sahni and colleagues indicate that this point could be identified ophthalmoscopically as the subthreshold (prethreshold) state for some eyes. Clearly, other eyes may pass through this International Classification of Retinopathy of Prematurity (ICROP) category to threshold disease, but remain unharmed. When is this line crossed and who will develop complications of ROP? A risk model (RM-ROP-2) can be used to identify infants with prethreshold disease and the greatest chance of adverse outcome (www.sph. uth.tmc.edu/rmrop/riskcalc/disclaimer. aspx). ${ }^{6}$ Additional analyses performed in the Early Treatment for Retinopathy of Prematurity Study (ETROP) indicated that ICROP findings are helpful in determining which infants with prethreshold disease should be treated earlier than at threshold. ${ }^{7}$ However, those molecular events, which result in the inexorable progression to complications of ROP, may begin before or even after the onset of these ICROP findings. ROP is thus a disease process whose molecular underpinnings resonate with but do not always exactly reflect physical findings. Today, therapeutic options must aim at ophthalmoscopically visible findings, but future medical and genetic therapies will target a broader and more nebulous line.

Br J Ophthalmol 2005;89:127-128.

doi: 10.1136/bjo.2004.058560

\section{Authors' affiliations}

W V Good, Smith-Kettlewell Eye Research Institute, San Francisco, CA, USA

R L Gendron, Memorial University, Newfoundland, Canada

Correspondence to: William V Good, MD, Smith Kettlewell Eye Research Institute, 2318 Fillmore Street, San Francisco, CA 94115, USA; Good@ski.org

\section{REFERENCES}

1 Hellstrom A, Perruzzi C, Ju M, et al. Low IGF-I suppresses VEGF-survival signaling in retinal endothelial cells: direct correlation with clinical retinopathy of prematurity. Proc Natl Acad Sci USA $2001 ; 98: 5804-8$.

2 Ozaki H, Yu AY, Della N, et al. Hypoxia inducible factor-lalpha is increased in ischemic retina: temporal and spatial correlation with VEGF expression. Invest Ophthalmol Vis Sci 1999;40:182-9.

3 Jeong JW, Bae MK, Ahn MY, et al. Regulation and destabilization of HIF- 1 alpha by ARD1mediated acetylation. Cell 2002;111:709-20.

4 Gendron RL, Good WV, Adams LC, et al. Expression of tubedown- 1 is suppressed in retinal neovascularization of proliferative diabetic retinopathy. Invest Ophthalmol Vis Sci 2001;42:3000-7.

5 Wall DS, Gendron RL, Good WV, et al. Conditional knockdown of tubedown-1 in endothelial cells leads to neovascular retinopathy. Invest Ophthalmol Vis Sci 2004 (in press).

6 Hardy RJ, Palmer EA, Dobson V, et al. Risk analysis of prethreshold ROP. Arch Ophthalmol 2003;121:1699-701.

7 Early Treatment for Retinopathy of Prematurity Cooperative Group. Revised indications for the treatment of retinopathy of prematurity: results of the early treatment for retinopathy of prematurity randomized trial. Arch Ophthalmol

2003;121:1684-96. 